\title{
An Electromagnetic Tracker System for the design of a dental superstructure
}

\author{
António H.J. Moreira ${ }^{1,2,3}$, Sandro Queirós ${ }^{1}$, Nuno F. Rodrigues ${ }^{1,3}$, ACM Pinho ${ }^{4}$, Jaime C. \\ Fonseca $^{2}$, João L. Vilaça ${ }^{1,3}$ \\ ${ }^{1}$ ICVS/3B's - PT Government Associate Laboratory, School of Health Sciences, Braga/Guimarães, Portugal \\ ${ }^{2}$ Algoritmi Center, School of Engineering, University of Minho, Guimarães, Portugal \\ ${ }^{3}$ DIGARC - Polytechnic Institute of Cávado and Ave, Barcelos, Portugal \\ ${ }^{4}$ Mechanical Department, University of Minho, Guimarães, Portugal
}

\begin{abstract}
Nowadays, different techniques are available for manufacturing full-arch implant-supported prosthesis, many of them based on an impression procedure. Nevertheless, the long-term success of the prosthesis is highly influenced by the accuracy during such process, being affected by factors such as the impression material, implant position, angulation and depth. This paper investigates the feasibility of a 3D electromagnetic motion tracking system as an acquisition method for modeling such prosthesis. To this extent, we propose an implant acquisition method at the patient mouth, using a specific prototyped tool coupled with a tracker sensor, and a set of calibration procedures (for distortion correction and tool calibration), that ultimately obtains combined measurements of the implant's position and angulation, and eliminating the use of any impression material. However, in the particular case of the evaluated tracking system, the order of magnitude of the obtained errors invalidates its use for this specific application.
\end{abstract}

\section{INTRODUCTION}

The medical treatment of partial or complete dental replacement has been significantly improved after the diffusion of osseointegrated implants to support dental prosthesis (Branemark et al. 1986). Different techniques are available today for manufacturing and assembling these biomechanical systems, typically based on a bridge structure, supported by a series of titanium screws implanted into the edentulous patient's jaw (Fig. 1a) (Lee et al. 2008).

Many dental techniques describe the use of different polymer printing materials (Lee et al. 2008) to measure and transfer the tridimensional information (position and orientation) of the patient's implants. Every step in the production of an implantsupported prosthesis influences the fit between the implants and the final prosthesis. One of the most critical steps for the long-term success of the prosthesis is the accuracy during the impression procedure (Lee et al. 2008; Al-Bakri et al. 2007), which is affected by factors such as the impression material, implant position, angulation and depth, as well as by difficulties in accessing the oral cavity, the variety of bone properties and its irregular geometrical features (Assuncao et al. 2004; Assunção et al. 2010; Sorrentino et al. 2010).

In general, the microgap (vertical, horizontal or angular, see Fig. 1b) misfit between implant components has been associated with several complications from both mechanical and biological origins. Furthermore, even though singular implant misfits are often imperceptible, the combination of several misfits in different implants may raise complications and compromise the entire prosthesis viability. A common consequence of misfits is screw loosening, which leads to structure instability and implant and/or screw fracture, and soft and/or hard tissue reactions due to increased dental plaque accumulation (Goodacre et al. 2003; Wang et al. 2002; Sahin et al. 2002; Eckert et al. 2000). Ultimately, such complications may demand prosthesis repair and, in some cases, its complete replacement for a new prosthesis.

Despite the development of novel and more precise fabrication methods, obtaining an absolute passive fit is practically impossible, especially in complete or partially edentulous patients. However, in most cases, small misfit tolerances (under $150 \mu \mathrm{m}$ ) are accepted, given that these do not lead to future implant complications (S Sahin \& Cehreli 2001; Kan et al. 1999).

The main goal of this study is to assess the feasibility of a 3D electromagnetic motion tracking system as an acquisition method for modeling fullarch implant-supported prosthesis. To this extent, we propose an implant acquisition method at the patient mouth based on a 3D electromagnetic tracker, and a set of calibration procedures, that obtains combined measurements of implant's position and angulation, excluding the need for impression material. The development of this system encompasses four main 

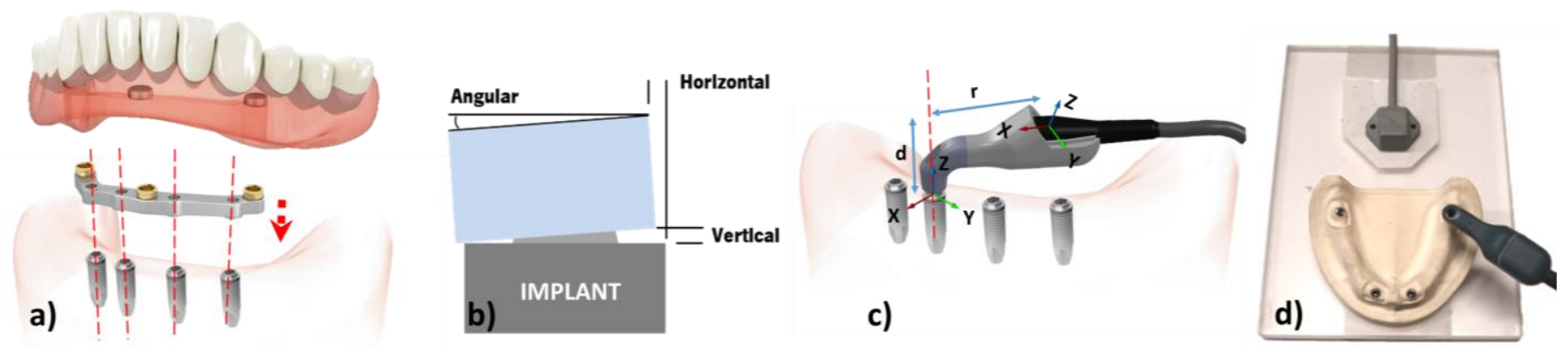

Figure 1. Prototype system overview. a) Implants and corresponding dental superstructure; b) Different types of microgap misfit (angular, horizontal and vertical); c) Implants' acquisition with electromagnetic tracker tool; d) Cast of human mandible (with 4 implants) used for evaluation.

steps: i) development of a specific tool designed to couple an electromagnetic sensor to an implant; ii) volume calibration to decrease electromagnetic distortions in the tracker readings (both position and orientation), namely from nearby metals, such as the titanium in the implants; iii) tool calibration to describe the rigid transformation from the sensor location to the tool's tip in order to assess the true implants' position; and iv) evaluate the stress distribution associated with the modeled prosthesis misfit using finite element analysis.

The potential advantages of this system are the reduction of the acquisition time of the patient jaw model, increased accuracy, reduction of the dependency of the entire procedure on the dentist experience, elimination of the need for physical transportation to the prosthesis centers and, thereby, eliminating the possibility of impression errors due to transport.

\section{METHODS}

\subsection{Prototype tool and system setup}

The proposed system requires the development and manufacturing of a miniature customized tool that couples an electromagnetic sensor to a dental implant (Fig. 2). In this step, a tool was prototyped in Accura Bluestone nanocomposite, to ensure stability and dimensional accuracy, while also being free of electromagnetic interference. This tool was designed to accommodate in one end an abutment with an internal hexagon connection and on the other end an eye-drop sensor from a Polhemus Liberty electromagnetic motion tracking system. Due to the irregular shape of the eye-drop sensor, it was fixed in the tool with an epoxy resin to ensure its stability (Fig. $2 b)$. The tool curved shape design was preferred to

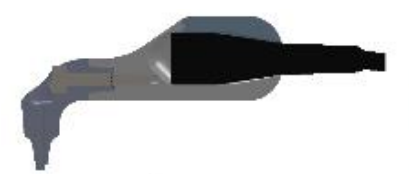

a)

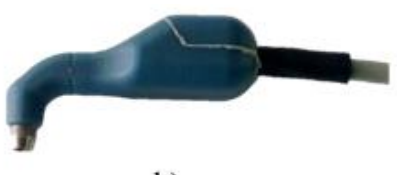

b)
Figure 2. Prototype tool for implant acquisition; a) cross section; b) final assemble. allow a more easy acquisition of the innermost implants.

In order to assess the reliability of the electromagnetic tracker for the implants' position and orientation acquisition, the tracker was calibrated in a laboratory environment. An industrial $6 \mathrm{DOF}$ robot (ABB IRB140, with $30 \mu \mathrm{m}$ of repeatability) was used to manipulate the tool in a 300x300x300mm volume. Since the robot and the Polhemus system have two different coordinate systems, it is paramount to align the two systems and remove the offsets between them prior to subsequent steps In this sense, a rigid registration between the two coordinate spaces is always performed, through an iterative closest point algorithm (ICP) (Rusinkiewicz \& Levoy 2001).

\subsection{Calibration methods}

This section presents the employed calibration methods and it's supporting mathematical principles. Suppose that a tracker sensor is moved freely inside a volume forming a cube in the true space. Let $\left\{p_{1}^{\prime}, \ldots, p_{m}^{\prime}\right\}$ denote the true tracker sensor position (measured by the industrial robot) and $\left\{p_{1}, \ldots, p_{m}\right\}$ the corresponding tracked measure ones (influenced by the field distortions). For each point $p_{i}$, the location error position vector (defined as the spatial difference between the true and measured position) can be computed as $v_{i}=p_{i}^{\prime}-p_{i}$. Similarly, let $\left\{q_{1}^{\prime}, \ldots, q_{m}^{\prime}\right\}$ denote the true tracker sensor orientations (expressed as quaternions) at points $p_{i}$ and $\left\{q_{1}, \ldots, q_{m}\right\}$ the corresponding measured ones. For each location, the error orientation quaternion is computed using quaternion composition:

$$
q_{\text {error }, i}=q_{i}^{\prime} \cdot q_{i}^{*}
$$

where $*$ represents the quaternion conjugate.

The calibration process will thus consist in extracting the information from the known error vectors (from the reference volume) to correct new unknown sensor data. In this sense, a correction vector can be found and applied to the unknown distorted measurement in order to approximate it to its true value. Note that our reference volume consists in a set of scattered points in a $3 \mathrm{D}$ volume (due to distortions), thus presenting no structure or order between their relative positions. 


\subsubsection{Position Correction}

Hardy's multiquadric (HMQ) interpolation algorithm (Hardy 1971; Zachmann 1997; Kindratenko 2000) is a global interpolation method that comes from the field of topography, with excellent results over scattered data in one or more dimensions. According to its formulation, the true value of a sensor data point, $p^{\prime}$, is given by:

$$
p^{\prime}=f(p)=\sum_{j=1}^{m} \alpha_{j} \cdot \omega_{j}(p)
$$

where

$$
\omega_{j}(p)=\sqrt{\left(p-p_{j}\right)^{2}+R^{2}}
$$

and $\mathrm{R}$ is the shape parameter controlling the interpolation and $\alpha_{j}$ are the expansion coefficient vectors $(x, y$ and $z)$.

In order to correct new sensor values, the unknown expansion coefficients need to be determined a priori. To this end, the reference volume points, $p_{i}^{\prime}$, and their corresponding sensor points, $p_{i}$, can be used to create a system of linear equations, easily expressed in the matrix form $\boldsymbol{W} \cdot \boldsymbol{A}=\boldsymbol{q}$ as:

$$
\left[\begin{array}{ccccc}
\omega_{1}\left(p_{1}\right) & \cdots & \omega_{j}\left(p_{1}\right) & \cdots & \omega_{m}\left(p_{1}\right) \\
\vdots & \vdots & \vdots & \vdots & \vdots \\
\omega_{1}\left(p_{i}\right) & \cdots & \omega_{j}\left(p_{i}\right) & \cdots & \omega_{m}\left(p_{i}\right) \\
\vdots & \vdots & \vdots & \vdots & \vdots \\
\omega_{1}\left(p_{m}\right) & \cdots & \omega_{j}\left(p_{m}\right) & \cdots & \omega_{m}\left(p_{m}\right)
\end{array}\right]\left[\begin{array}{c}
\alpha_{1} \\
\vdots \\
\alpha_{j} \\
\vdots \\
\alpha_{m}
\end{array}\right]=\left[\begin{array}{c}
p_{1}^{\prime} \\
\vdots \\
p_{i}^{\prime} \\
\vdots \\
p_{m}^{\prime}
\end{array}\right]
$$

By solving such system, the required expansion coefficients are found and any uncorrected sensor value, $p$, can be then compensated using equation (2).

\subsubsection{Orientation correction}

The correction of orientation errors, represented using quaternions, involves the construction of an interpolating or approximating function. To this end, we start by determining in which tetrahedron the measured location lies. For this, we use a Delaunay tetrahedralization (Hagedorn et al. 2007), which provides substantial advantages, namely the fact that data does not need to be collected on a grid, and thus scattered points can be used. This enables the collection of data points more densely in regions of greater distortion.

To interpolate the error quaternion from the values stored within the tetrahedron vertices, the system computes the barycentric coordinates of the measured location relative to that tetrahedron (Borst 2004). Subsequently, the orientation correction $\left(d_{q}\right)$ for the measured point is determined by a weighted average of the data at the tetrahedrons vertices, using the previous barycentric coordinates as weights $\left(w_{i}\right)$ :

$$
d_{q}=\operatorname{Normalize}\left(\sum_{i=0}^{3} w_{i} \cdot q_{\text {error }, i}\right)
$$

Finally, a calibrated tracker reading can be computed from a sensor reading, $q$, by:

$$
q^{\prime}=d_{q} \cdot q
$$

\subsection{Tool Calibration}

In order to assess the true implants' position based on the calibrated sensor readings, a rigid transformation $(T)$ describing the position and orientation correction from the sensor's location to the tool's tip is required to be computed. To this end, a calibration process must be performed to assess the tool transformation parameters, namely: a) the tool radius $(r) ; b)$ the orientation quaternion $\left(q_{\text {tool }}\right)$ representing the transformation from the sensor's axes to the tool's axes, $O\left(q_{\text {tool }}\right)$; and c) the tool offset in the z-axis $(d)$, see Figure 1c. The final tool transformation is thus given by:

$$
T=T_{z}(-d) \cdot T_{x}(r) \cdot O\left(q_{\text {tool }}\right)
$$

For such calibration, the proposed setup encompasses one cast with four titanium implants (DIO Implants) with external hexagon connections. In each implant, 6 positions are acquired using the electromagnetic motion tracking system with the prototype tool (Fig. 1d) and used to estimate the tool parameters.

First, to estimate the tool radius ( $r)$, a circle was fitted to each set of 6 position (per implant). This was accomplished by initially fitting a plane, by means of singular value decomposition (SVD), and extracting the plane's normal vector. Inside this plane, the Newton-Taubin circle fit method (Taubin 1991) was used to find the circle central point and corresponding radius.

At this point, the orientation quaternion can be estimated based on the orientation of the initial points (given by the tracker and calibrated according to section 2.2.2) and the tool axes (given by the plane's normal, the vector from the point's position to the circle central point and the corresponding orthogonal vector). The rotation quaternion obtained for each implant in the cast is thus given by the average of the quaternions for the acquired 6 points.

At last, the tool offset in the z-axis, $d$, is estimated fitting the true implant's orientation vector from the ground truth $\left(\vec{v}_{g}\right)$ and the previous obtained planes' normal $\left(\vec{v}_{p}\right)$ - Figure 3 . To this end, a line segment is

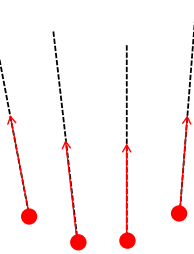

a)

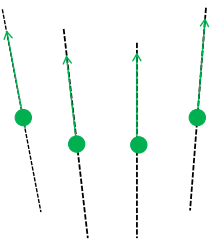

b)

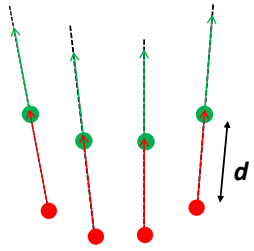

c)
Figure 3. Estimation of the tool offset in the z-axis. a) Points and orientation vector (red) from the ground truth; b) Circles center points and planes' normal (green) from radius estimation; and c) estimated fit between line segments (black dotted lines) using combined ICP and GPS. The offset $(d)$ is given by the Euclidian distance between points. 
obtained for each vector (4 from ground truth and 4 from the planes' normal) by sampling $n$ points along the vector with step $h$. Then, the ICP algorithm is used to fit both set of points (ground truth against real ones). In order to minimize errors associated with the choice of the parameters $n$ and $h$, a generalized pattern search (GSP) (Kolda et al. 2003) algorithm is used, in combination with the ICP algorithm, by minimizing the following cost function:

$$
\begin{array}{rl}
S(n, h)=k_{a} I & C P(n, h) \\
& +k_{b} \sum_{i=1}^{4} \cos ^{-1}\left(\vec{v}_{p} \cdot \vec{v}_{g}\right) \\
& +k_{c} \sigma\left(\left[d_{1} \cdots d_{4}\right]\right)
\end{array}
$$

where $k_{a}$ is the weight for the ICP error term, $k_{b}$ is the weight for the sum of angle errors between real and ground truth orientations (after fitting) and $k_{c}$ the weight for the standard deviation of the estimated offsets, $d_{i}$. The weights were chosen to give the same importance for all terms. Such cost function was designed to find the result that minimizes the angles deviation and the variation of the computed offset among implants. Note that such minimization strategy is possible as long as the 4 vectors are not parallel to each other (which is the case of the designed cast).

\subsection{Finite element method}

A computerized three-dimensional finite-element model (FEM) of a full-arch fixed dental implant prosthesis with 4 supports was created from the ground truth points. The model consisted in a curved beam, following a spline curve defined by the 4 implants with $94.0 \mathrm{~mm}$ long, $3.0 \mathrm{~mm}$ high, and 5.0 $\mathrm{mm}$ wide. The implant supports (abutments) were defined as cylinders with $4.7 \mathrm{~mm}$ diameter, with an inner hole of $1.9 \mathrm{~mm}$ diameter. A titanium alloy was selected as the prosthesis material.

In order to perform the stress distribution analysis, a finite element analysis (FEA) software, COMSOL Multiphysics 4.3, was used.

\section{EXPERIMENTS}

In order to assess the performance of the proposed acquisition system, three evaluation tests were performed: a) accuracy assessment of the proposed calibration algorithm used to minimize tracker's distortions; b) evaluation of the tool calibration step using a cast of human mandible with 4 implants, comprising the acquisition of the cast implants with a coordinate measurement machine (CMM - WENZEL XOrbit) with $3 \mu \mathrm{m}$ accuracy as ground truth; and c) a validation test comprising a FEA of the stress distribution along the prosthesis due to displacements between implants and prosthesis implant supports.

\subsection{Distortion error minimization}

The proposed tool (Section 2.1) was fixed at an extremity of a carbon-fiber $10 \times 10 \mathrm{~mm}$ square tube with $600 \mathrm{~mm}$ length, and with the opposite extremity fixed on an industrial 6 DOF robot (ABB IRB140, with $30 \mu \mathrm{m}$ of repeatability) (Fig. 4). The described system was used to acquire samples inside the $300 \times 300 \times 300 \mathrm{~mm}$ volume (with $30 \mathrm{~mm}$ step, in a total of 1331 points) to be used as references for the calibration process. For each point of the volume, 1000 samples from the electromagnetic tracker were acquired and averaged. Complementing the volume points, 30 validation points were also acquired to validate the calibration performance.

Using the calibration methods presented in Section 2.2 , the average error and its standard deviation was computed for both position and orientation.

\subsection{Tool calibration errors}

Using the calibration method presented in Section 2.3 , the variability of the transformation parameters $\left(r, q_{\text {tool }}\right.$ and $\left.d\right)$ was assessed among the 4 implants in the calibration cast.

\subsection{Finite Element Analysis}

For the final validation test, a new set of 4 readings were acquired with the electromagnetic tracker (one per implant). Using the average transformation parameters to create the rigid transformation for tool calibration, the implant's position and orientation were estimated and the magnitude of misfit computed for the 4 implants.

Moreover, the full-arch prosthesis model was created afterwards in SolidWorks 2013 and exported to COMSOL to perform FEA (von Mises stress). The final mesh consists of 217726 domain elements, 24686 boundary elements, and 2047 edge elements. In order to perform the stress analysis, the model was restrained at one end implant and the remaining implants' supports displaced along the $(x, y, z)$ axes to fit in the ground truth positions.

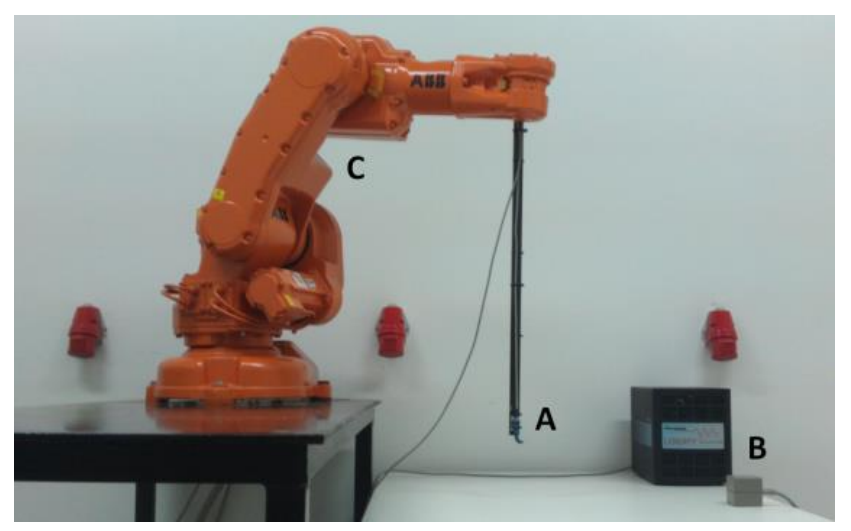

Figure 4. System setup - A) prototype tool; B) Polhemus electromagnetic tracker and C) robot ABB IRB140. 


\section{RESULTS AND DISCUSSION}

In regard to the accuracy assessment of the proposed position calibration algorithm (Section 2.2.1), the results (calibrated and non-calibrated) for the average error and standard deviation in each axis, and their combined magnitude, are summarized in Table 1.

Table 1. Calibration performance (mean error and standard deviation for each axis and combined magnitude) for the real volume data acquired with the Polhemus tracker system.

\begin{tabular}{lllll}
\hline \multirow{2}{*}{ Calibration } & Magnitude & $\mathrm{X}$ & $\mathrm{Y}$ & $\mathrm{Z}$ \\
\cline { 2 - 5 } & $\mathrm{mm}$ & $\mathrm{mm}$ & $\mathrm{mm}$ & $\mathrm{mm}$ \\
\hline None & $4.03 \pm 2.44$ & $1.11 \pm 0.99$ & $2.47 \pm 2.20$ & $2.27 \pm 2.01$ \\
HMQ & $0.53 \pm 0.25$ & $0.15 \pm 0.14$ & $0.47 \pm 0.26$ & $0.11 \pm 0.09$ \\
\hline
\end{tabular}

The Hardy's multiquadric interpolation algorithm presented major improvements when compared to the initial error (no calibration), therefore significantly reducing the errors of the sensor's readings.

Regarding the orientation correction (Section 2.2.2), the average angular error, and corresponding standard deviation, is presented in Table 2, prior and after calibration.

Table 2. Orientation calibration performance (mean angular error and standard deviation) obtained with the acquired volume data.

\begin{tabular}{ll}
\hline Calibration & Angle $\left({ }^{\circ}, \mu \pm \sigma\right)$ \\
\hline None & $5.60 \pm 0.69$ \\
(Borst) & $0.06 \pm 0.02$ \\
\hline
\end{tabular}

As in the previous case, the calibration process significantly reduced the errors, diminishing the effects of the electromagnetic distortions in the following steps of the proposed system.

In what concerns the tool calibration, the transformation parameters obtained for each implant (A to D), and the average values computed and used for the subsequent stress analysis, are summarized in Table 3.

Table 3. Tool calibration performance (the transformation parameters, $r, q_{\text {tool }}$ and $d$, for each of the 4 implants, and the resulting average value).

\begin{tabular}{|c|c|c|c|c|c|}
\hline & \multicolumn{4}{|l|}{ Implant } & \multirow{2}{*}{$\mu \pm \sigma$} \\
\hline & A & B & $\mathrm{C}$ & $\mathrm{D}$ & \\
\hline \multirow[t]{3}{*}{$r(\mathrm{~mm})$} & 31.969 & 31.958 & 31.950 & 31.953 & $31.958 \pm 0.009$ \\
\hline & 0.997 & {$[0.997]$} & 0.997 & 0.996 & {$[0.997]$} \\
\hline & 0.039 & 0.046 & 0.048 & 0.057 & 0.045 \\
\hline \multirow[t]{2}{*}{$q_{\text {tool }}$} & -0.044 & -0.044 & -0.049 & -0.058 & -0.046 \\
\hline & 0.051 & 0.048 & 0.048 & 0.049 & 0.049 \\
\hline$d$ & 18.438 & 18.402 & 18.411 & 18.426 & $18.420 \pm 0.016$ \\
\hline
\end{tabular}

According to these results, a variability around $10 \mu \mathrm{m}$ was obtained for the tool radius. Regarding the orientation quaternion, similar values were found among the implants. Finally, for the tool offset in the $\mathrm{z}$-axis, a standard deviation of near $16 \mu \mathrm{m}$ was found. Such errors were associated with an angular average error of $0.25 \pm 0.13^{\circ}$ between the implant's normal (measured by the prototype tool) and the ground truth vector (measured by the CMM) after the ICP algorithm. Together, is important to note that the combination of the different parameters in one single rigid transformation encompasses an increased combined error, which may ultimately preclude the usage of such system.

The aforementioned possibility was tested in a final validation test to measure the implant's misfit, as well as the stress distribution, whose results are presented in Table 4 and Figure 5.

Table 4. Validation test misfit (measured as the Euclidean distance between the ground truth position and the obtained with the proposed tracker system).

\begin{tabular}{lllll}
\hline & Implant A & Implant B & Implant C & Implant D \\
\cline { 2 - 5 } & $\mathrm{mm}$ & $\mathrm{mm}$ & $\mathrm{mm}$ & $\mathrm{mm}$ \\
\hline Misfit & 0.3515 & 0.2976 & 0.3238 & 0.3646 \\
\hline
\end{tabular}

In this last test, the system produced misfits around $300 \mu \mathrm{m}$. Moreover, the FEA software presented a maximum stress of $7700 \mathrm{MPa}$, with higher stresses around the implants' supports, as expected. Moreover, it was found that the stress increase is proportional to the increase of the misfit and also higher in implants farther to the electromagnetic emitter. Overall, the presented results show that the proposed system has errors above the application requirements, precluding its current usage in the daily practice.

\section{CONCLUSION}

This work presents a study to assess the feasibility of a 3D electromagnetic tracking system as an acquisition method of implant's position for modeling full-arch implant-supported prosthesis. The potential advantages of this system are the reduction of the acquisition time of the patient jaw model, increased accuracy, and reduction of the dependency of the entire procedure on the dentist experience.

To this extent, a specific tool was prototyped to couple the Polhemus tracker to an implant, allowing the acquisition directly from the patient's mouth.

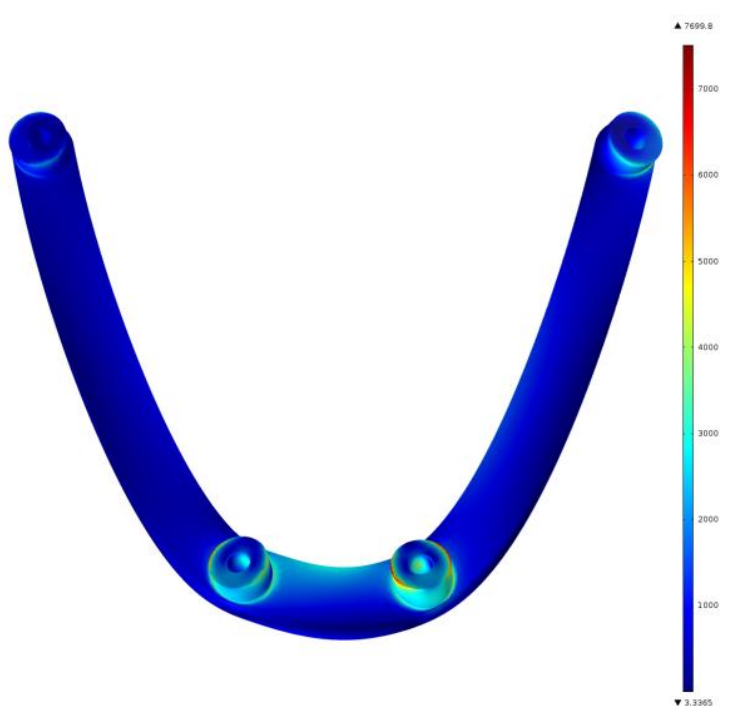

Figure 5. von Mises stress distribution obtained with finite element analysis software for the full-arch prosthesis modelled during the validation test. 
Moreover, calibration methods (for both position and orientation) were presented to reduce electromagnetic distortions in the sensor's reading, with significant improvements (under $0.53 \mathrm{~mm}$ and $0.06^{\circ}$ ). Subsequently, a tool calibration procedure was conceived to transform the sensor's position to the tool's tip. Finally, a validation test was performed to assess the implant's misfit and the stress distribution in the full-arch prosthesis.

Overall, the order of magnitude of the obtained errors invalidates the use of this system for the fullarch modeling of implant-supported prosthesis. The most probable reason for this impossibility relies not in the calibration procedures, but rather on the physical limitations of the tracking device, in particular its sensitivity to magnetic interferences.

Nevertheless, we believe there is an opportunity for this kind of tracking systems to be used in other medical applications with less stringent requirements, such as surgical instrument navigation, prosthesis accurate placement, physical therapy and rehabilitation, and kinesiology studies.

As future work, we intend to identify different principles and sensors for the accurate acquisition of the implant's positions and angulations, such as mechanical or optical systems.

\section{ACKNOWLEDGMENTS}

This work has been supported by FCT - Fundação para a Ciência e Tecnologia in the scope of the Ph.D. grant SFRH/BD/68270/2010 and the project EXPL/BBB-BMD/2146/2013.

\section{REFERENCES}

Al-Bakri IA, Hussey D, Al-Omari WM. 2007. The dimensional accuracy of four impression techniques with the use of addition silicone impression materials. J Clin Dent; 18:29-33.

Assunção WG, Britto RC, Ricardo Barão VA, Delben JA, dos Santos PH. 2010. Evaluation of impression accuracy for implant at various angulations. Implant Dent; 19:167-74.

Assuncao WG, Filho HG, Zaniquelli O. 2004. Evaluation of transfer impressions for osseointegrated implants at various angulations. Implant Dent; 13:358-66.

Borst CW. Tracker calibration using tetrahedral mesh and tricubic spline models of warp. In: IEEE Virtual Real 2004; p. 19-26.

Branemark P-I, Zarb GA, Albrektsson T, Rosen HM. 1986. Tissue-integrated prostheses. osseointegration in clinical dentistry. Plast Reconstr Surg. 77:496-497.

Eckert SE, Meraw SJ, Cal E, Ow RK. 2000. Analysis of incidence and associated factors with fractured implants: a retrospective study. Int J Oral Maxillofac Implants. 15:662-7.
Goodacre CJ, Bernal G, Rungcharassaeng K, Kan JYK. 2003. Clinical complications in fixed prosthodontics. J Prosthet Dent; 90:31-41.

Hagedorn JG, Satterfield SG, Kelso JT, Austin W, Terrill JE, Peskin AP. 2007. Correction of Location and Orientation Errors in Electromagnetic Motion Tracking. Presence Teleoperators Virtual Environ. 16:352-366.

Hardy RL. 1971. Multiquadric equations of topography and other irregular surfaces. J Geophys Res; 76:1905-1915.

Kan JY, Rungcharassaeng K, Bohsali K, Goodacre CJ, Lang BR. 1999. Clinical methods for evaluating implant framework fit. J Prosthet Dent; 81:7-13.

Kindratenko V V. 2000. A survey of electromagnetic position tracker calibration techniques. Virtual Real. 5:169-182.

Kolda TG, Lewis RM, Torczon V. 2003. Optimization by Direct Search: New Perspectives on Some Classical and Modern Methods. SIAM Rev; 45:385-482.

Lee H, So JS, Hochstedler JL, Ercoli C. 2008. The accuracy of implant impressions: a systematic review. J Prosthet Dent; 100:285-91.

Rusinkiewicz S, Levoy M. 2001. Efficient variants of the ICP algorithm. In: Proc Third Int Conf 3-D Digit Imaging Model [Internet]. [place unknown]: IEEE Comput. Soc; [cited 2014 Jan 20]; p. 145-152.

Sahin S, Cehreli MC, Yalçin E. 2002. The influence of functional forces on the biomechanics of implant-supported prostheses--a review. J Dent; 30:271-82.

Sahin S, Cehreli MC. 2001. The significance of passive framework fit in implant prosthodontics: current status. Implant Dent; 10:85-92.

Sorrentino R, Gherlone EF, Calesini G, Zarone F. 2010. Effect of implant angulation, connection length, and impression material on the dimensional accuracy of implant impressions: an in vitro comparative study. Clin Implant Dent Relat Res; 12 Suppl 1:e63-76.

Taubin G. 1991. Estimation of planar curves, surfaces, and nonplanar space curves defined by implicit equations with applications to edge and range image segmentation. IEEE Trans Pattern Anal Mach Intell; 13:1115-1138.

Wang T-M, Leu L-J, Wang J, Lin L-D. 2002. Effects of prosthesis materials and prosthesis splinting on peri-implant bone stress around implants in poor-quality bone: a numeric analysis. Int J Oral Maxillofac Implants; 17:231-7.

Zachmann G. 1997. Distortion correction of magnetic fields for position tracking. In: Proc Comput Graph Int: IEEE Comput. Soc. Press; p. 213-220. 